\title{
Numerical Simulation of Steel Fiber's Crack Resistance on Cement-based Composites
}

\author{
Chen Jiahao*, Xu Zhihong, Zhang Yong, Zhu Kangwei \\ School of Science, Nanjing University of Science and Technolgy, Nanjing, China \\ Email address: \\ 1506928995@qq.com (Chen Jiahao) \\ ${ }^{*}$ Corresponding author
}

To cite this article:

Chen Jiahao, Xu Zhihong, Zhang Yong, Zhu Kangwei. Numerical Simulation of Steel Fiber's Crack Resistance on Cement-based Composites. Science Discovery. Vol. 8, No. 6, 2020, pp. 139-145. doi: 10.11648/j.sd.20200806.14

Received: September 20, 2020; Accepted: October 23, 2020; Published: November 4, 2020

\begin{abstract}
In this paper, the cement base and steel fiber are modeled separately through a separate model. The cement base uses a concrete damage plastic model; while the steel fiber imports the programmed program into the simulation software to generate randomly distributed steel fibers, and the steel fiber uses a truss unit. After the separation model is modeled, it is combined with the extended finite element to simulate the cracks of the steel fiber reinforced cement-based beam with I-shaped notch under the action of three-point bending, and the crack propagation until the cement-based beam is completely broken. According to the simulation results, the load-deflection and load-crack opening displacement curves of the cement base were drawn; the crack evolution process was tracked; various fracture parameters were calculated; and the stress time history curves of the steel fibers in different regions were drawn. The results show that: steel fiber can effectively improve the fracture energy and fracture toughness of the cement base, and play a toughening and crack resistance effect on the cement base; the crack resistance of steel fiber is mainly produced by the steel fiber in the middle span and the crack area. The steel fibers at both ends of the beam have little effect on the cement base.
\end{abstract}

Keywords: Steel Fiber, Cement-based, Extended Finite Element Method, Fracture Process, Fracture Parameter

\section{钢纤维对水泥基复合材料阻裂作用的数值模拟}

陈嘉浩", 徐志洪, 张勇, 朱康伟

南京理工大学理学院, 南京, 中国

邮箱

1506928995@qq.com(陈嘉浩)

摘要：本文通过分离式模型对水泥基和钢纤维分别建模，水泥基采用混凝土损伤塑性模型；而钢纤维则是将编程编写 完的程序导入仿真软件中，生成随机分布的钢纤维，钢纤维采用桁架单元。分离式模型建模完成以后，将其与扩展有 限元结合, 模拟了含I形切口的钢纤维增强水泥基梁在三点弯作用下出现裂缝, 裂缝扩展直至水泥基梁完全断裂的整个 过程。根据仿真结果绘制了水泥基的荷载-挠度、荷载-裂缝口张开位移曲线; 追踪了裂纹演化过程; 计算了各项断裂 参数; 并绘制了不同区域钢纤维的应力时程曲线。结果表明：钢纤维能有效地提高水泥基的断裂能和断裂韧度，对水 泥基起到增韧阻裂作用; 钢纤维的阻裂作用主要是由跨中和裂缝区域的钢纤维产生的, 梁两端的钢纤维对水泥基的影 响很小。

关键词: 钢纤维, 水泥基, 扩展有限元, 断裂过程, 断裂参数 


\section{1. 引言}

混凝土是目前应用最广泛的材料之一, 它的一个突出 问题就是易开裂和脆性断裂 [1-4]。为了提高混凝土的性能, 钢纤维混凝土应运而生 [5]。钢纤维的存在能够大大地提升 混凝土的延性 [6-9], 混凝土在受力开裂以后, 钢纤维与混 凝土之间的粘结滑移以及钢纤维的阻裂作用, 阻碍和延缓 混凝土的开裂。

国内外对钢纤维水泥基的数值仿真基本可以分为两类: 整体式模型和分离式模型。整体式模型认为钢纤维在水泥基 中是均匀分布的, 钢纤维与水泥基之间没有相互作用, 而是 作为一个整体来承担外界的作用。整体式模型需要对水泥基 的本构关系进行修改来模拟钢纤维水泥基的属性。因为整体 式模型无需在生成钢纤维, 因此也无法单独把钢纤维提取出 来研究其对水泥基的增强作用。分离式模型则需要对水泥基 和钢纤维分别建模, 水泥基多采用混凝土损伤塑性模型或者 弥散开裂模型; 而钢纤维则多是将编程导入仿真软件中, 生 成随机分布的钢纤维。钢纤维通常采用梁单元或者桁架单元。 本文将采用分离式模型对钢纤维水泥基进行仿真。

扩展有限元法 (Extended Finite Element Method, XFEM) 是处理非连续计算问题的一种数值模拟方法, 将非连续的 位移引入ABAQUS中的位移表达式中[10-15], 以此来计算 裂纹两端的破坏及变形。和传统的有限元相比, XFEM计 算中产生的裂纹与计算的网格是彼此独立的, 在裂纹扩展 以后, 不需要对网格进行重新划分。因此, XFEM可以实 现裂缝任意扩展的仿真。本文结合粘聚力接触以及XFEM, 模拟了含 $\mathrm{I}$ 形切口的钢纤维增强水泥基梁在三点弯加载下 出现裂缝, 裂缝扩展直至混凝土梁完全断裂的整个过程。

\section{2. 钢纤维水泥基三点弯模型的建立}

\section{1.几何模型及网格划分}

本文采用二维模型, 平面外厚度设置为 $1 \mathrm{~mm}$ 。带切口 的水泥基梁三点弯加载模型包括水泥基、钢纤维以及垫块 三个部分。水泥基为 $40 \mathrm{~mm}$ 宽, $160 \mathrm{~mm}$ 长的带切口的长方体 模型, 长方体建立完成后, 在跨中切割出底边 $2 \mathrm{~mm}$, 高 $16 \mathrm{~mm}$
的I型切口。水泥基采用CPS4R线性减缩积分单元网格划分, 该网格划分方法适用于较规则的几何体, 计算速度较快。

研究表明, 在混凝土中能起到改性作用的钢纤维的长 径比为 $40-80$ 。因此, 本文采用直径 $0.3 \mathrm{~mm}$, 长度 $20 \mathrm{~mm}$ 的圆 直形钢纤维。将python撰写的编程导入到ABAQUS中, 批 量生成体积率为 $0.6 \%$ 钢纤维后, 对其进行 $\mathrm{T} 2 \mathrm{D} 2$ 网格划分。

垫块设置为刚体，无需考虑其材料及网格划分。本文 分别对钢纤维增强水泥基梁和普通的水泥基梁进行仿真, 以进行对比，装配好的模型如图1所示。

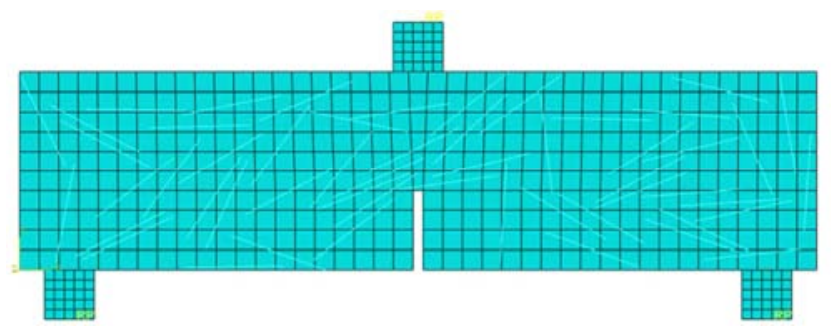

（a）钢纤维增强水泥基模型图

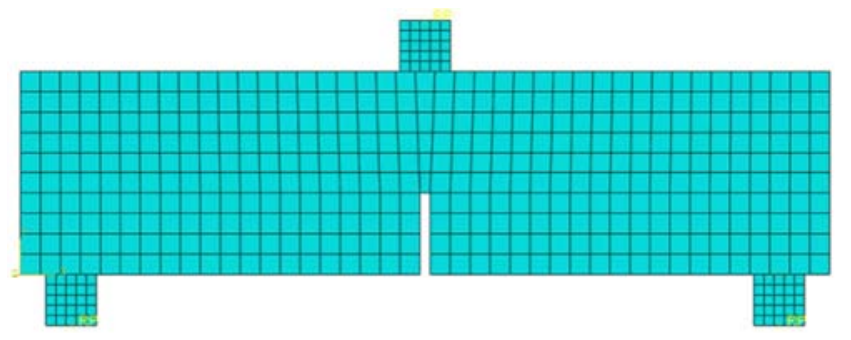

(b)普通水泥基模型图

图1 模型图。

\section{2.材料模型}

混凝土采用损伤塑性模型, 在设置损伤塑性模型的同 时设置XFEM的参数。传统上认为混凝土是脆性材料, 因 此XFEM裂缝的起裂准则采用最大主应力准则, 即裂纹尖 端的应力达到最大抗拉强度, 裂纹开展, 具体数据如表 1 所示。钢纤维参数如表2所示。

表1 混凝土参数。

\begin{tabular}{|c|c|c|c|c|}
\hline 名称 & 膨胀角 & 偏心率 & & $K$ \\
\hline 混凝土 & 36 & 1.16 & & 0.6667 \\
\hline \multicolumn{5}{|c|}{ 表2 钢纤维参数。 } \\
\hline 延性损伤 & & 弹性 & & \\
\hline 断裂应变 & 应力三轴度 & 杨氏模量/MPa & 泊松比 & \\
\hline 0.25 & 1.35 & 200000 & 0.3 & \\
\hline
\end{tabular}

\section{3.加载方式及分析部设置}

本文模拟了含切口的水泥基梁在三点弯加载下出现 裂缝, 裂缝扩展直至混凝土梁完全断裂的整个过程。钢纤 维以内置区域的形式与混凝土梁进行约束。三个刚体垫块 与混凝土梁之间设置摩擦接触, 摩擦系数为 0.3 。对下部
的两个垫块设置固定约束, 上部的垫块设置参考点, 通过 在参考点施加位移的形式对模型进行加载, 加载速度为 $1 \mathrm{~mm} / \mathrm{s}$ 。当水泥基完全断裂时, 仿真结束。

\section{3. 仿真结果分析}

\section{1. 钢纤维对荷载变形曲线的影响}

仿真得到的应力云图如图2、3所示，根据仿真结果提 取的荷载-挠度曲线如图4所示。 


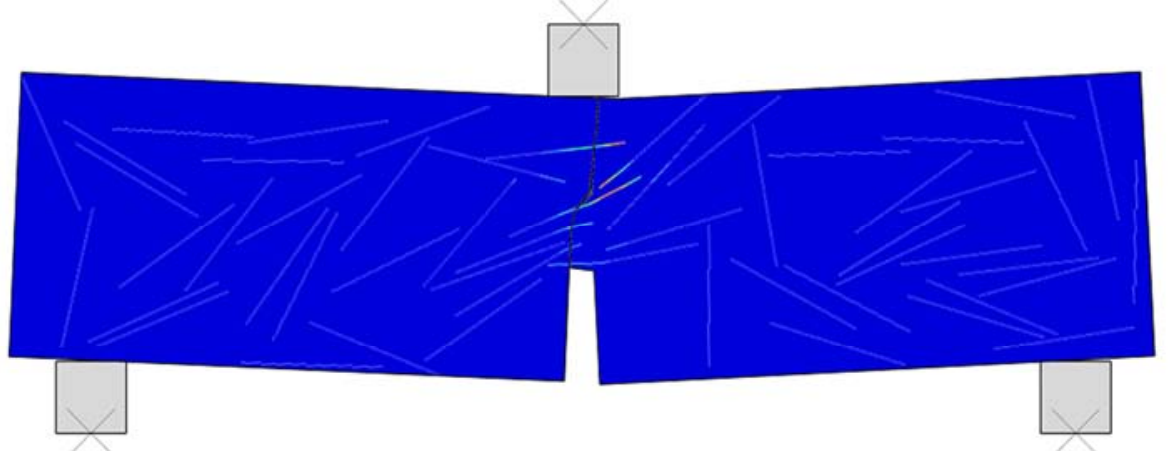

图2 钢纤维增强水泥基应力云图。

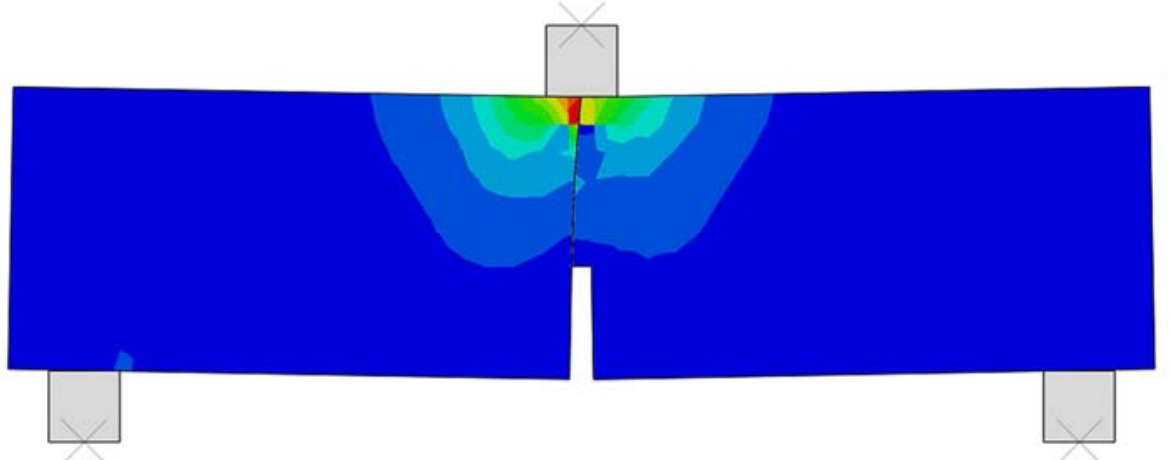

图3 普通水泥基应力云图。

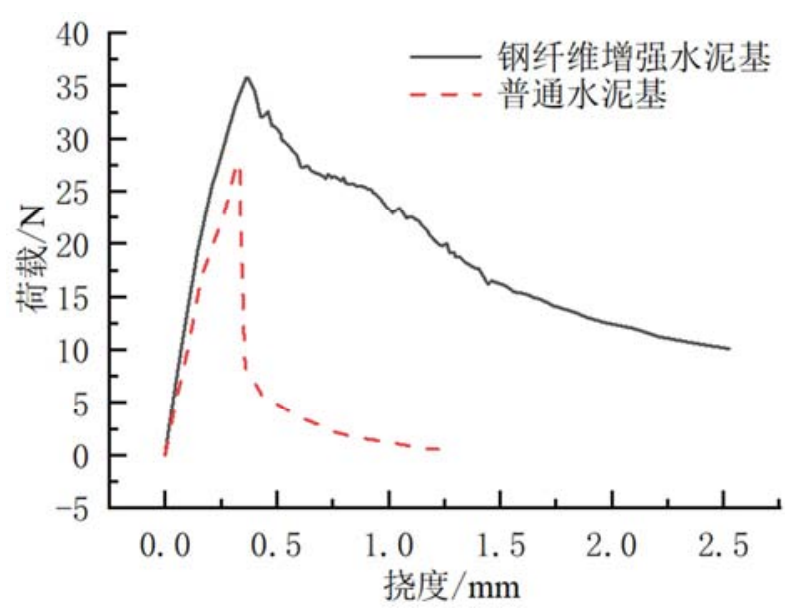

图4 荷载-挠度曲线。

普通水泥基和钢纤维增强水泥基的曲线呈现不同的 变化趋势。普通水泥基梁在加载初期, 曲线线性上升, 此 时水泥基处于弹性阶段。随着位移的继续增加, 水泥基产 生裂缝, 并且裂缝在产生以后急剧发展, 贯穿整个梁。水 泥基的承载能力急剧下降, 曲线呈直线下降趋势。荷载挠度曲线显现出了普通水泥基明显的脆性特点。

钢纤维混凝土梁的荷载-挠度曲线可以分为三个阶段。 弹性阶段: 此时水泥基还没有产生裂缝, 曲线呈线性增长。 开裂阶段: 随着荷载的增加, 直线开始转为曲线, 水泥基 内的微裂缝开始发展。与此同时, 跨越裂缝两端的钢纤维 产生桥联作用, 阻碍裂缝的扩展, 提高了水泥基的抗弯承
载能力。失稳破坏阶段: 当荷载达到峰值即失稳荷载以后, 裂缝的发展速度开始加快。混凝土逐渐退出工作, 更多的 钢纤维产生桥联作用, 并逐渐被拔出, 曲线呈缓慢下降趋 势。

普通水泥基与钢纤维增强水泥基的荷载-裂缝口张开 位移 (CMOD) 曲线如图5所示。

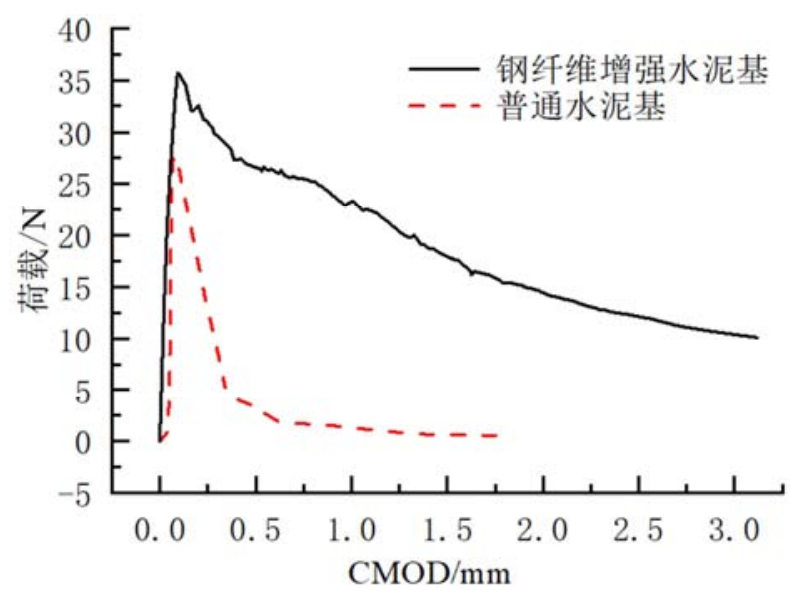

图5 荷载-裂缝口张开位移 (CMOD) 曲线。

在弹性阶段, 普通水泥基和钢纤维水泥基的裂缝张开 口位移均呈线性增长。此时裂缝张开口位移还较小, 如果 卸除载荷, 裂缝张开口位置几乎能完全恢复。到了开裂阶 段, 普通水泥基梁在开裂以后, 裂缝迅速发展, 发展为宏 观裂纹, 并迅速贯穿整个构件, 曲线急速下降。钢纤维增 
强水泥基在开裂后, 跨越裂缝的纤维传递应力, 使水泥基 梁保持平衡。随着裂缝张开口位移的继续增加, 钢纤维逐 渐被拔出, 曲线呈缓慢下架趋势, 钢纤维增强水泥基表现 出较好的延性。

从两个曲线图可以看出, 钢纤维增强水泥基的极限荷 载、挠度等都优于普通水泥基材料。裂缝产生后, 横跨裂
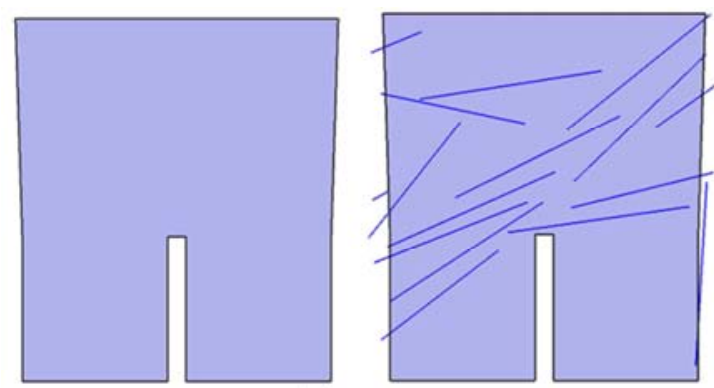

(a) step 1
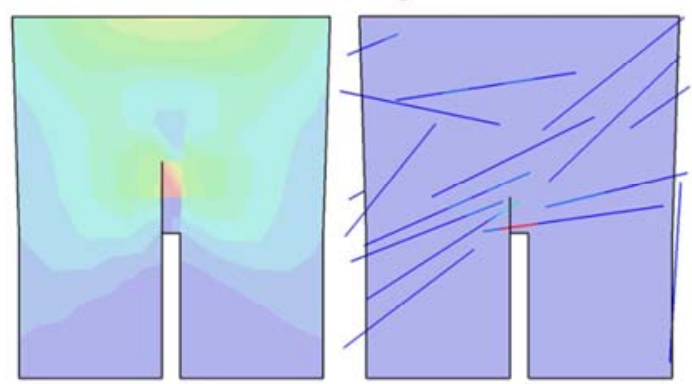

(c) step 3
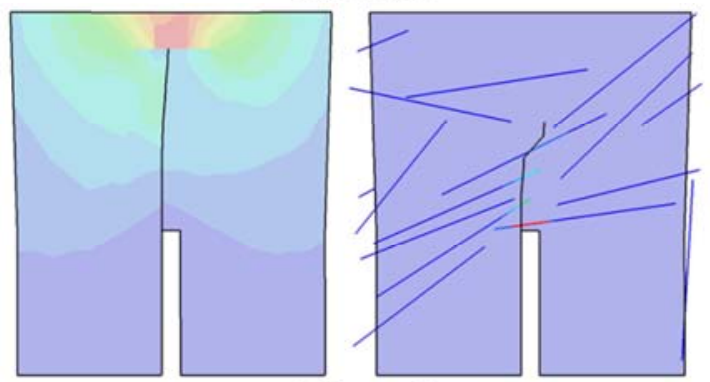

(e) step 5
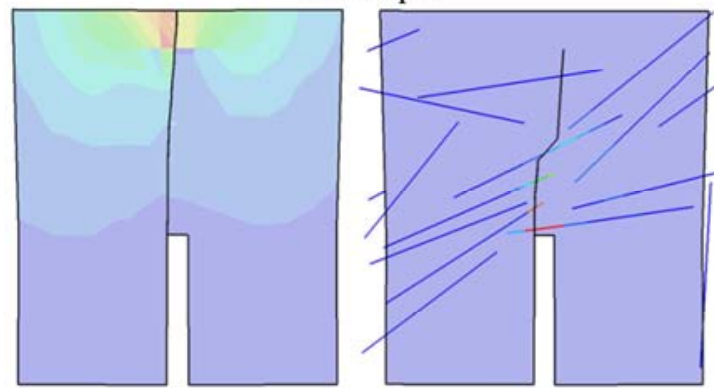

(g) $\operatorname{step} 7$
缝的钢纤维起到了桥联作用, 延缓和阻碍了裂缝的发展, 大大地提高了水泥基的韧性。

\section{2. 损伤演化分析}

普通水泥基和钢纤维水泥基在仿真过程中裂纹扩展 的过程如下图6(a)-(h)所示。其中左边的是普通水泥基的裂 纹扩展过程, 右边的是钢纤维水泥基的扩展过程。
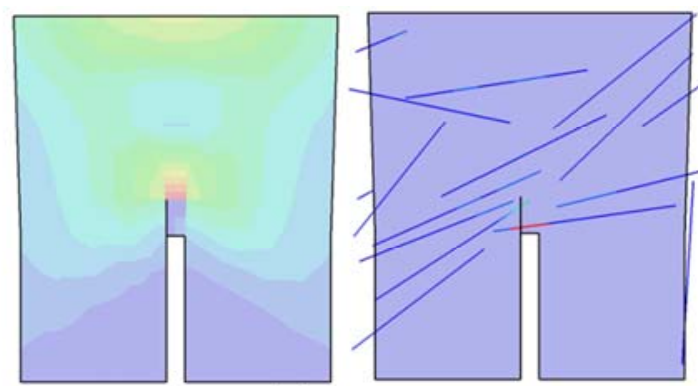

(b) $\operatorname{step} 2$

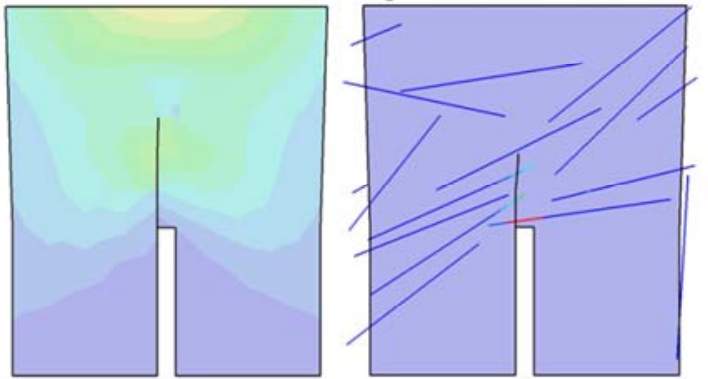

(d) step 4
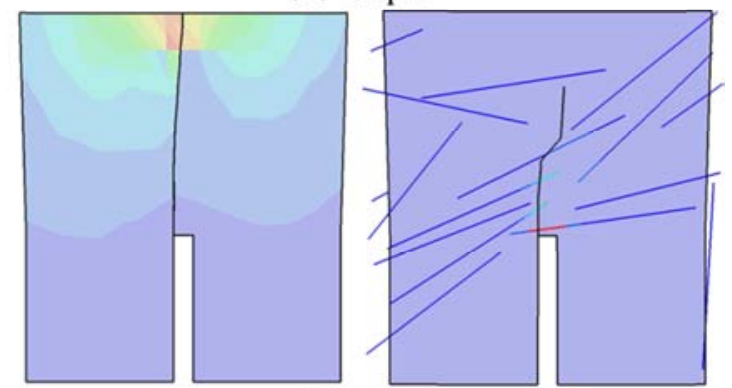

(f) $\operatorname{step} 6$
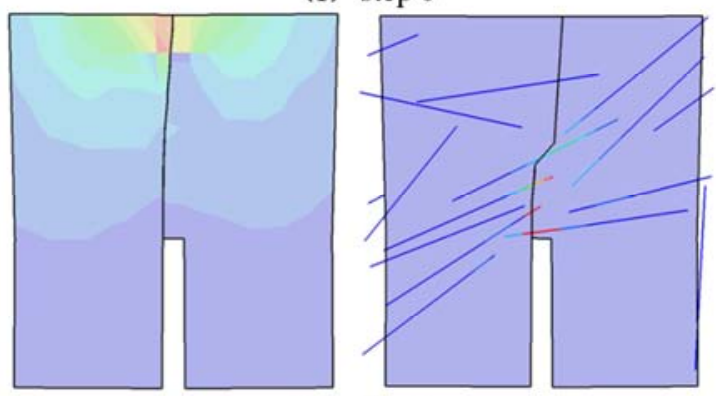

(h) step 8

图6 普通水泥基和钢纤维水泥基裂纹扩展图。

由于仿真的水泥基构件较小, 并预制有相对切口深度 为 0.4 的 $\mathrm{I}$ 型缺口, 因此两种水泥基构件均只出现了一条裂 纹。可以看出, 在相同的时间下, 普通水泥基的裂纹扩展 速度要远快于钢纤维水泥基。普通水泥基的裂纹在出现后, 就呈直线形式发展, 并在较短的时间内贯穿整个构件, 使
构件失去承载能力。钢纤维水泥基的每次裂纹扩展, 都会 使部分钢纤维横跨裂纹, 使钢纤维产生桥联作用, 承受荷 载, 这同时也减缓了裂纹的扩展速度。

钢纤维的存在还对裂纹的形态发展产生了较大的影 响。钢纤维水泥基的裂纹比普通水泥基的要更加曲折。为 
了定量地评估纤维对基体材料的影响, Hassan N A, Airey $\mathrm{G} \mathrm{D}$ 等人提出了裂纹曲折系数的概念。裂纹曲折系数的示 意图如图7所示。

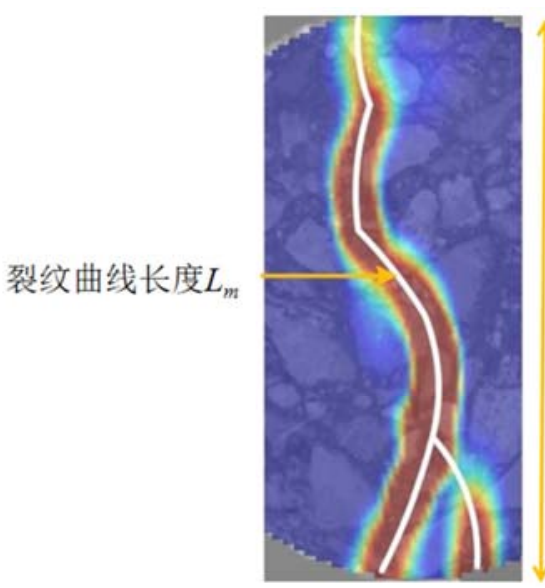

图7 裂纹曲折系数示意图。
裂纹曲折系数的计算公式为:

$$
\text { 裂纹曲折系数 }=\frac{\text { 裂纹曲线长度 } L_{m}}{\text { 裂纹投影长度 } L_{p}}
$$

普通水泥基和钢纤维水泥基的裂纹曲折系数计算结 果如表3所示。

表3 裂纹曲折系数。

\begin{tabular}{lll}
\hline 组别 & 普通水泥基 & 钢纤维水泥基 \\
\hline 裂纹投影长度 $L_{p} / \mathrm{mm}$ & 24 & 24 \\
裂纹曲线长度 $L_{m} / \mathrm{mm}$ & 24.079 & 25.008 \\
裂纹曲折系数 & 1.003 & 1.042 \\
\hline
\end{tabular}

与普通水泥基相比, 钢纤维水泥基的裂纹曲折系数有 所提高, 这说明钢纤维的存在延长了水泥基的开裂路径, 起到了阻裂的作用。

普通水泥基与钢纤维增强水泥基中水泥基受拉损伤 的情况如图8、9所示, 图中红色表示单元严重受拉损伤, 黄色和绿色表示单元受一定程度的受拉损伤。

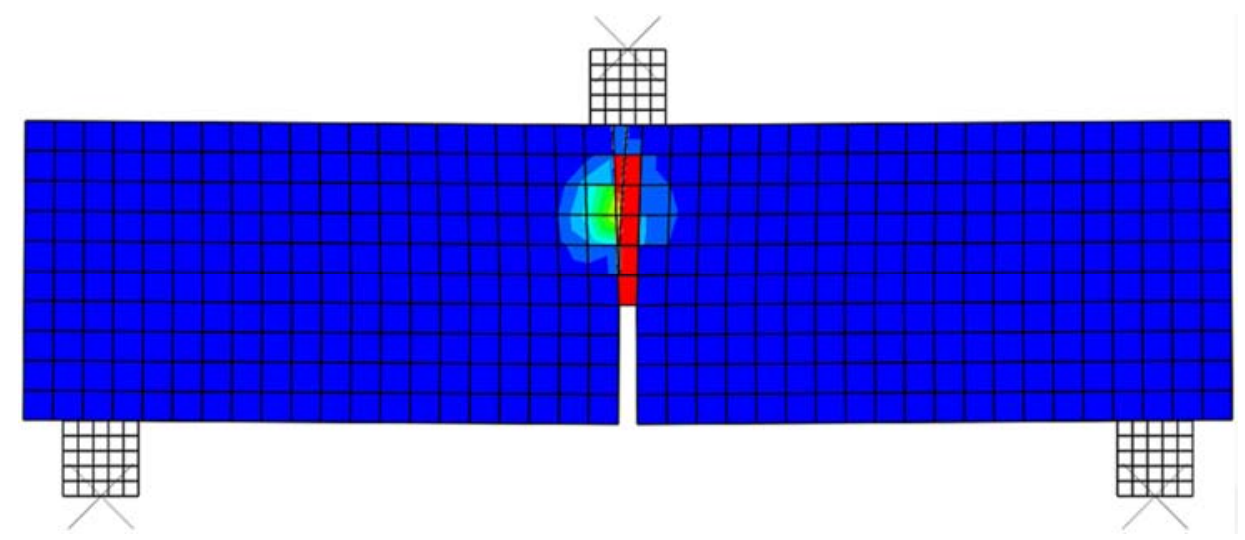

图8 普通水泥基单元受拉损伤图。

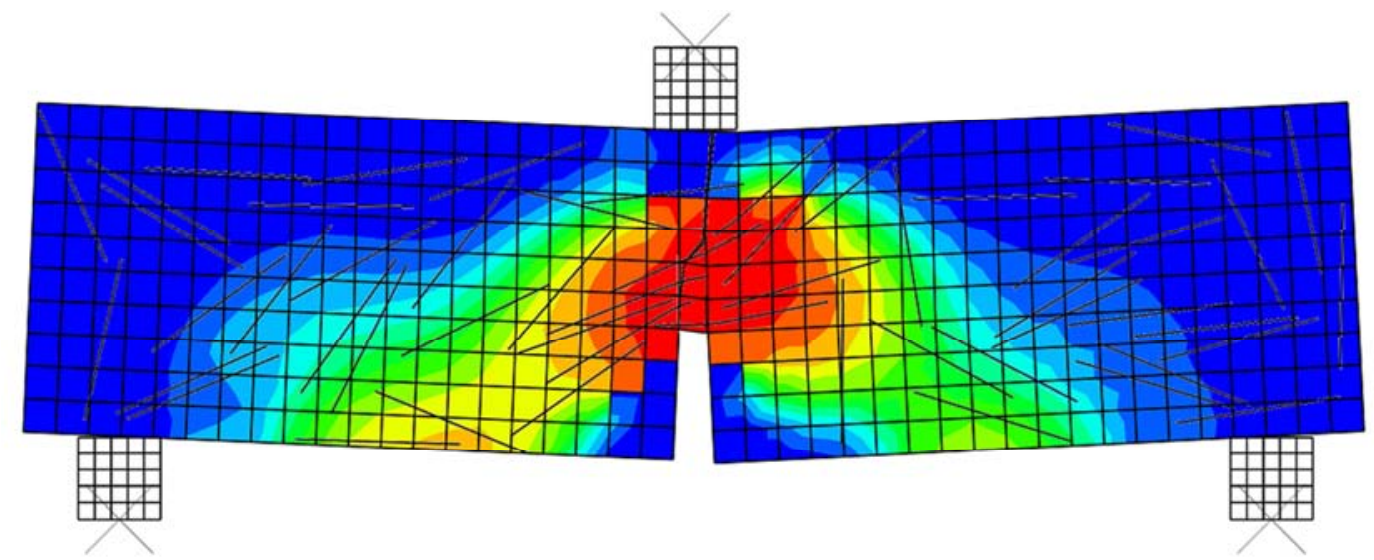

图9 钢纤维水泥基单元受拉损伤图。

由上图可知, 由于普通水泥基的裂纹一旦出现, 就会 很快贯穿整个构件, 失去承载能力。只有裂纹经过的单元 发挥了部分抗拉作用, 其余部分水泥基的抗拉作用几乎没 有发挥, 这表现出了水泥基明显的脆性特点。而钢纤维水 泥基的裂纹在产生后, 使横跨裂纹的钢纤维产生桥联作用, 钢纤维和两端水泥基的相互作用, 使得裂开的水泥基仍能
发挥出抗拉作用。同时，裂纹两端没有开裂的水泥基，在 荷载的作用下, 与钢纤维产生拉拔作用, 阻碍钢纤维被拔 出的同时，起到了抗拉的效果。钢纤维水泥基中起到抗拉 作用的水泥基单元要远远多于普通水泥基, 钢纤维对是水 泥基的增㓞作用非常明显。 


\section{3. 钢纤维对水泥基断裂特征参数的影响}

基于仿真结果, 本文提取了计算数据来具体评估钢纤 维对水泥基断裂性能的影响, 这里选用的断裂特征参数为 断裂能与断裂韧度。

断裂能指的是荷载将混凝土梁完全压断的整个过程 中, 裂缝扩展单位面积所需的能量。切口水泥基梁三点弯 作用下的断裂能计算公式如下:

$$
\begin{aligned}
& G_{F}=\frac{W_{0}+m g \delta_{0}}{A_{\text {lig }}} \\
& f\left(\frac{a}{W}\right)=2.9\left(\frac{a}{W}\right)^{1 / 2}-4.6\left(\frac{a}{W}\right)^{3 / 2}+21.8\left(\frac{a}{W}\right)^{5 / 2}-37.6\left(\frac{a}{W}\right)^{7 / 2}+38.7\left(\frac{a}{W}\right)^{9 / 2}
\end{aligned}
$$

式中, $F Q$ 为临界荷载; $S$ 为支座之间距离; $w$ 为试件 高度; $B$ 为试件厚度; $a$ 为预制裂缝的长度。

根据以上公式计算得到的断裂参数如表4所示。

表4 断裂特征参数。

\begin{tabular}{lll}
\hline 组别 & 普通水泥基 & 钢纤维水泥基 \\
\hline 极限荷载 $/ \mathrm{N}$ & 27.798 & 35.708 \\
临界裂缝张口位移 $/ \mathrm{mm}$ & 0.070 & 0.096 \\
断裂能 $/ \mathrm{N} \cdot \mathrm{mm}-1$ & 0.330 & 2.221 \\
断裂韧度 $/ \mathrm{kN} \cdot \mathrm{m}^{-3 / 2}$ & 964 & 1238 \\
\hline
\end{tabular}

式中, $W 0$ 为荷载-挠度曲线围成的面积; $\mathrm{m}$ 为支座间 混凝土梁的质量; $\delta 0$ 为混凝土梁的最大挠度; Alig为断裂 韧带的面积。

断裂韧度指的是混凝土阻止裂缝扩展的能力, 本文采 用美国材料与试验协会 (ASTM) 建议的断裂韧度计算公 式。断裂韧度的计算公式如下:

$$
K_{I C}=\frac{F_{Q} S}{B W^{3 / 2}} f\left(\frac{a}{W}\right)
$$

钢纤维增强水泥基的极限荷载、临界张开口位移、断 裂能以及断裂韧度都优于普通水泥基, 尤其是断裂能, 是 普通水泥基的 6.73 倍。这是因为钢纤维的阻碍了裂缝的快 速发展，使得水泥基在开裂后仍有一定的延性，提高了水 泥基的韧性，改善了水泥基一裂即断的问题。

\section{4. 不同位置钢纤维阻裂效果的分析}

仿真得到的钢纤维水泥基梁中钢纤维的应力云图如 图10所示,

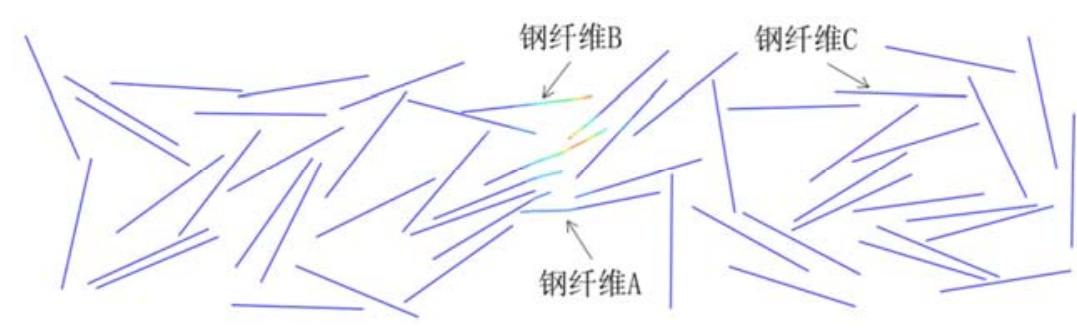

图10 钢纤维应力云图。

观察图10钢纤维的应力云图, 发现不同位置的钢纤维 应力差别很大。跨中部位的钢纤维应力普遍较大, 而梁两 端的钢纤维则应力很小。分别选取跨中底部的钢纤维A、 跨中顶部的钢纤维 $\mathrm{B}$ 以及梁端部钢纤维 $\mathrm{C}$ 的 3 根钢纤维, 3 根纤维的位置如图2（b）所示。分别取A、B钢纤维中横 跨裂缝的一个网格单元, 以及钢纤维 $C$ 的一个单元, 绘制 这些单元的应力-水泥基挠度曲线如图11所示。

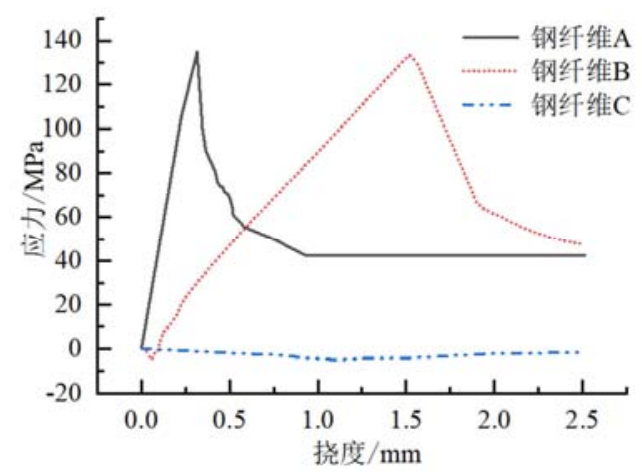

图11 钢纤维应力-水泥基挠度曲线。
钢纤维A在荷载加载初期便处于受拉区。加载初期, 混凝土还处于弹性阶段, 没有产生裂缝, 钢纤维应力增长 缓慢。随着荷载变大, 混凝土开始产生微裂纹, 钢纤维上 的应力增长加快。当混凝土产生宏观裂纹后, 位于裂缝两 端的钢纤维开始起到桥联作用, 钢纤维上的应力突然加大。 荷载作用下, 裂缝继续扩张, 钢纤维与混凝土基体之间产 生相对滑移, 钢纤维的应力达到峰值以后开始逐渐变小。 最后, 钢纤维的应力趋于平稳, 该应力为钢纤维与混凝土 基体之间的残余应力。

钢纤维B中的钢纤维在加载初期处于受压区, 钢纤维 产生较小的压应力。在荷载作用下, 混凝土产生裂缝并扩 张, 混凝土梁的中性轴逐渐向梁顶部移动, 原本的受压区 逐渐变为受拉区。当裂缝扩展到原本的受压区时, 原本受 压的钢纤维横跨裂缝的两端产生桥联作用。钢纤维的压应 力突然转变为拉应力。很快, 裂缝贯穿了水泥基梁, 钢纤 维B处的裂缝宽度逐渐增大, 钢纤维的应力也因此增大, 到达峰值应力后钢纤维与水泥基逐渐脱粘, 钢纤维应力也 因此减小。但还没等到裂缝宽度大到足以使钢纤维与水泥 基之间完全脱粘，仿真就结束了。 
钢纤维 $C$ 为梁端部的钢纤维上的单元应力, 由曲线可 知, 在整个加载过程中, 钢纤维上的应力都很小, 完全没 有发挥出钢纤维的抗拉和桥联作用。

与实验相比, 数值仿真可以直观地研究混凝土内部钢 纤维的应力。根据不同位置钢纤维的应力时程曲线, 可以 看出, 位于跨中以及裂缝区域内的钢纤维在水泥基开裂以 后产生桥联作用, 阻碍裂缝的发展, 增加了混凝土的韧性。 而梁两端的钢纤维应力很小, 几乎没有发挥出作用。在工 程实际中, 根据钢纤维的受力情况合理地布置钢纤维, 可 以有效地节约工程成本, 提升钢纤维的使用效率。

\section{4. 结论}

本文结合python和扩展有限元, 模拟了含切口的钢纤 维增强水泥基梁在三点弯加载下出现裂缝, 裂缝扩展直至 混凝土梁完全断裂的整个过程。根据仿真结果得到了以下 几点认识:

（1）钢纤维增强水泥基的荷载-挠度曲线以及荷载裂缝口张开位移曲线都优于普通水泥基, 钢纤维大大地提 高了水泥基的韧度。

(2) 钢纤维的桥联作用使裂纹两端的水泥基产生抗 拉作用, 减缓了裂纹的扩展速度。

（3）钢纤维增强水泥基的各项断裂特征参数均优于 普通水泥基, 尤其是断裂能, 是普通水泥基的6.73倍。

（4）钢纤维的阻裂作用主要是由跨中和裂缝区域的 钢纤维产生的, 梁两端的钢纤维对水泥基的影响很小。

\section{参考文献}

[1] 陈肇元, 崔京浩, 朱金铨.钢筋混凝土裂缝机理与控制措施 [J].工程力学, 2006,23 (01) : 86-107.

[2] 任晓丹, 李杰. 基于损伤理论的钢筋混凝土结构裂缝分析 [J]. 同济大学学报, 2015,43（8）：1129-1134.
[3] 童谷生, 胡宗棋, 徐鹏华. 尖锐 $V$ 型切口混凝土梁的应力强 度因子研究 $[J]$.应用数学和力学, 2018,39(03):300-310.

[4] 傅喻, 陈有亮. 相对切口深度对混凝土断裂性能影响试验研 究[J].水资源与水工程学报, 2017,28(04):187-192.

[5] 薛建锋, 沈培辉, 王晓鸣. 混凝土材料的正交异性侵彻深度 模型[J].南京理工大学学报, 2016,40(4):381-385.

[6] Belletti B, Cerioni R, Meda A,. Design Aspects on Steel Fiber-Reinforced Concrete Pavements [J] Journal of Materials in Civil Engineering,20(9)2008,599-607.

[7] Hyun-Oh Shin,Kyung-Hwan Min,Denis Mitchell.Confinement of ultra-high- performance fiber reinforced concrete columns[J]. Composite Structures,2017,176.

[8] 邱继生. 钢纤维混凝土结构非线性有限元分析方法研究 [J]. 混凝土, 2011,3: 17-20.

[9] Benard sojeh, S.M.ASCE 1; Fatigue Behavior of Steel FiberConcrete in Direct Tension[J]American Society of Civil Engineers.2017, 68-97.

[10] 孙亚飞, 耿飞, 高培伟,等.掺聚丙烯纤维泡沫混凝土性能与 仿真分析[J].南京理工大学学报, 2016,40(4):504-505.

[11] 刘永胜, 王肖钧, 金挺.钢纤维混凝土力学性能和本构关系 研究[J].中国科学技术大学学报, 2007,(07):717-723.

[12] 李华伟, 王文达.ABAQUS二次开发在钢管混凝土结构有限 元分析的应用[J].建筑结构学报, 2013,34(8):353-358.

[13] 王力.钢纤维-混凝土基体界面粘结性能数值分析 [J].水利与 建筑工程学报, 2019,17(2):56-60.

[14] 夏冬桃, 吴吴, 熊思慧, 等.混杂纤维增强高性能混凝土断 裂能研究 $[J]$.混凝土, 2020,6:113-119.

[15] 田稳苓, 刘博雄, 卿龙邦.钢纤维定向对水泥基复合材料等 效断裂韧度的影响 [J].混凝土, 2018,7:58-61. 